\title{
Práticas de Aprendizagem e Metodologias do Ensino numa Licenciatura em Dança
}

\author{
Maria João Alves \\ Faculdade de Motricidade Humana, Universidade de Lisboa \\ Instituto de Etnomusicologia - Centro de Estudos em Música e Dança (INET-md) \\ E-mail: mjalves@fmh.ulisboa.pt \\ Margarida Moura \\ Faculdade de Motricidade Humana, Universidade de Lisboa \\ Instituto de Etnomusicologia - Centro de Estudos em Música e Dança (INET-md) \\ E-mail: mmoura@fmh.ulisboa.pt
}

\section{Resumo}

As características da dança, como atividade humana natural e vinculada a determinada cultura, são essenciais para encararmos as funções e o contexto nos quais as práticas de aprendizagem na licenciatura em dança estão inseridas, nomeadamente ao nível das metodologias de ensino da dança. Neste artigo procedemos a uma fundamentação teórica das atividades que preconizamos com os nossos estudantes-professores na unidade curricular de Metodologia do Ensino da Dança, pertencente ao $3^{\circ}$ ano da licenciatura em dança na Faculdade de Motricidade Humana, Universidade de Lisboa. De modo genérico, propomos aos estudantes a consolidação e a amplificação do repertório de comportamento de ensino e o desenvolvimento, a implementação e a avaliação do seu projeto de ensino-aprendizagem de dança. Usando uma abordagem focada em oferecer enquadramento e espaço de aplicação de práticas com diferentes métodos, estilos e estratégias de ensino em diferenciados contextos educativos, a unidade curricular procura uma articulação criteriosa dos variados constructos teóricos, mediando os estudantes a capacitarem-se com um poder crítico e a conhecer quem vai adquirir o quê e de que forma, como propõe Pinto (2011). Explicitamos processos de construção de currículo, explanando princípios de atuação que preconizamos como relevantes ao exercício da função docente, especificando competências dos estudantes-professores e vinculando-nos à teoria do ciclo de aprendizagem experiencial (KOLB, 1984) ao propor uma atuação dinâmica entre planificação - intervenção - controlo - avaliação - reformulação da atividade pedagógica.

\section{Palavras-chave}

Ensino de dança. Metodologias de ensino.

Planificação. Prática pedagógica. Eansino superior português.
Dance characteristics, as a natural human activity and linked to a certain culture, are essential to consider the functions and context in which the learning practices in the dance undergraduate course are integrated, namely regarding dance teaching methodologies. In this paper, we present a theoretical framework for the activities that we recommend for our student-teachers in this curricular unit of Methodology of Dance Teaching, part of the 3rd year of the dance studies degree at the Human Kinetics Faculty, University of Lisbon. In general, we propose that students consolidate and amplify their repertoire of teaching behavior and develop, implement and evaluate their dance teaching-learning project. Using an approach focused on offering a framework and space for the application of practices with different teaching methods, styles, and strategies in different educational contexts, the curricular unit pursues a careful articulation of the diverse theoretical constructs, mediating students to empower themselves with critical empowerment and to know who will acquire what and in what way, as proposed by Pinto (2011). We make explicit processes of curricular construction, explaining principles of action that we advocate as relevant to the exercise of the teaching function, specifying competencies of student-teachers and linking ourselves to the theory of experiential learning cycle (KOLB, 1984) by proposing a dynamic action between planning - intervention - control - evaluation - reformulation of pedagogical activity.

\section{Keywords}

Dance teaching. Teaching methodologies.

Planning. Pedagogical practice. Portuguese higher education. 


\section{Introdução}

A Licenciatura em Dança da Faculdade de Motricidade Humana da Universidade de Lisboa (FMH-ULisboa) existe há 33 anos, desde 1988, tendo como principal finalidade formar especialistas com uma visão interdisciplinar e sistémica dos problemas específicos ao desenvolvimento da dança em contexto de educação formal, informal e não formal. Considera, assim, adequados conhecimentos no domínio das técnicas de dança, das técnicas expressivas, das técnicas de animação, das técnicas somáticas de bem-estar e das tecnologias fundamentais de comunicação e informação. Do seu currículo consta um conjunto diversificado de abordagens no domínio da Dança, das Ciências da Motricidade e das Ciências da Educação que sustentam e fundamentam o ensino e a aprendizagem da dança enquanto objeto de estudo. Dessa diversidade formativa singulariza-se a unidade curricular, Metodologia do Ensino da Dança (MED), enquanto disciplina terminal de formação do licenciado em dança.

De natureza clínica e aplicativa, a unidade curricular de MED trata os conteúdos recebidos e trabaIhados ao longo da formação superior em dança, na perspetiva do planear, do executar, do controlar e do avaliar o exercício laboratorial de prática pedagógica: a planificação pedagógica, o plano de aula, os métodos de instrução, as estratégias comunicacionais, os conteúdos programáticos, as atividades de aprendizagem, a avaliação de desempenho e os relatórios das sessões e das unidades de ensino da dança e do movimento expressivo. Centra-se na aprendizagem do aluno utilizando como principais modelos de trabalho, o modelo de aprendizagem por objetivos (SACRISTÁN, 1990) - da pedagogia do conteúdo à pedagogia da aprendizagem através de aprendizagens comportamentais e performances a curto prazo - e o modelo de aprendizagem por projeto (DEWEY, 1978) - pedagogia do diálogo e da autonomia centrado na resolução de problemas numa sequência de operações entre a preparação, a execução e a apreciação/divulgação/avaliação do projeto.
MED assume como principal objetivo a aquisição de conhecimentos, de competências e de habilidades profissionais, específicas ao ensino da dança, na assunção de que estas permitem utilizar a dança e o movimento expressivo como um meio privilegiado de desenvolvimento e educação. Contribui, decididamente, para a formação de professores de dança, que realizam a sua atividade profissional com populações diversificadas (crianças, jovens, adultos, ou seniores) e em contextos educativos diferenciados - escolar e comunitário (educativo e de animação e lazer), sejam escolas públicas, privadas, autarquias, estúdios de dança, companhias de dança, entre outras instituições, que direta ou indiretamente estão relacionadas com o fenómeno da dança ao serviço da educação. Com este tipo de abordagem o estudante-professor em formação aumenta e diversifica o seu nível de conhecimentos, bem como melhora o padrão de desempenho pedagógico fundamental à sua intervenção profissional.

\section{- O professor como mediador de aprendizagem}

Preconizamos que o estudante da licenciatura em dança fique ciente das necessidades e diferenças dos alunos e dotado de conhecimentos, habilidades e atitudes de modo a ser capaz de mediar eficazmente a aprendizagem (WARBURTON, 2008). Para o efeito utiliza e ajusta estratégias de ensino, meios e recursos pedagógicos que se adequem a vários propósitos criando um ambiente de aprendizagem desafiante, numa variedade de contextos educativos.

A abordagem que desenvolvemos assenta no conceito de professor eficaz "aquele que maximiza as aquisições dos alunos, agindo de acordo com um conjunto explícito de princípios sequenciais, coerentes e relevantes num contexto educacional específico"' (COLE; CHAN, 1986, p. 64 apud KILLEN, 2016, p. 2, tradução nossa). Para atribuir ordem, coerência e relevân-

1 "effective teacher is one who maximises the achievements of students' by acting 'in accordance with an explicit set of principles that have order, coherence and relevance in the particular instructional context". 
cia ao contexto de instrução de MED delineamos e aplicamos o que consideramos serem os princípios de atuação, adequados e relevantes ao exercício da função docente, como sejam:

- Compromisso com a aprendizagem. Realçam-se e operacionalizam-se conceitos e práticas assentes em aprendizagens provenientes de diferentes unidades curriculares da licenciatura em dança que tratam as questões da análise do processo de ensino-aprendizagem, do controlo e aprendizagem motora e dos fundamentos e práticas de intervenção em dança.

- Integridade no processo. Enfatizam-se percursos de ação que estabelecem conexões, identificam padrões, e organizam parcelas de conhecimento, comportamento e ação num todo que se quer significativo.

- Respeito pelas capacidades dos estudantes. Estimula-se que os estudantes-professores apliquem conhecimentos adquiridos aquando da resolução de problemas, bem como, comuniquem e relacionem conhecimentos anteriores com os resultantes das aprendizagens em MED, questionando, analisando e sintetizando informação. Contribui-se para que os alunos apresentem e desenvolvam o que Nightingale e O'Neil (1994) definem como competências características da aprendizagem de alta qualidade.

$\mathrm{Na}$ pedagogia por objetivos, o ensino da dança assenta na eficácia e eficiência pedagógicas, na qualidade do professor de dança (conceito de professor eficaz) que queremos diligenciar com os nossos estudantes-professores. Definir objetivos de aprendizagem gerais e comportamentais, específicos e operacionais, estratégias de comunicação, métodos/estilos de intervenção e avaliação do processo de ensino e de aprendizagem constitui, estrutura e caracteriza a pedagogia das aprendizagens comportamentais, das performances a curto prazo e das tarefas concretas a realizar pelos alunos. Os objetivos estipulados afiguram-se observáveis, medíveis e alcançáveis, sendo o objetivo final a soma dos objetivos parcelares e intermédios, pelo que a eficiência pedagógica assenta num processo de ensino ordenado, sequencial e com um desenho operacional preciso (SACRISTÁN, 1990).

Por sua vez o trabalho de projeto, verdadeira pedagogia do diálogo e da autonomia, com elevado valor socioafetivo e cognitivo na construção do saber (VASCONCELOS, 2011), favorece a interdisciplinaridade, como abordagem de ensino e pesquisa, e contribui para dar significado e intencionalidade às aprendizagens através do envolvimento dos seus participantes na resolução de problemas concretos e na transferência de saberes para novas aprendizagens. Potencia experiências anteriores proporcionando assim um aprimoramento e o desenvolvimento das próprias competências como instrumentos de aprendizagem e compreensão da realidade. Vasconcelos (2011) destaca, ainda, o desenvolvimento de competências essenciais numa sociedade do conhecimento, como sejam, a recolha e tratamento de informação e, simultaneamente, a aprendizagem do trabalho de grupo, a colaboração, a tomada de decisão negociada, a atividade metacognitiva, e o espírito de iniciativa e criatividade. O estudante deixa de ser um passivo recetor de saberes dos outros para se assumir como investigador e criador ativo de saberes, fundamental aos fins sociais da educação.

Neste tipo de abordagem o papel do aluno torna-se ativo, voluntário, empenhado, responsável, comprometido afetivamente com as tarefas e metodólogo de si mesmo. Por sua vez, o papel do docente assume-se como clarificador dos objetivos, provocador do problema, regulador, dinamizador, animador, gestor, mediador e avaliador.

\section{Abordagem à aprendizagem experiencial}

A originalidade de MED reside precisamente no confronto dos estudantes com a planificação, realização e controlo da atividade pedagógica em contexto real de intervenção, seja com uma população alvo (crianças, jovens ou seniores) escolhida por eles ou proposta pelos docentes. Trabalha-se a função 
docente, garantindo a autonomia do aluno nos aspetos considerados fundamentais para a intervenção profissional em contextos educativos - formais e informais, do licenciado em dança, como sejam:

- Domínio de técnicas, estratégias de comunicação e modelos de intervenção em ensino e aprendizagem de dança que favorecem a eficácia pedagógica;

- Prática de formas de dança diversificadas e de modelos expressivos diferenciados, que possam ser conducentes ao conhecimento didático do conteúdo e à sua adequada seleção;

- Destreza na aplicação de técnicas de observação e de supervisão pedagógicas;

- Conhecimento e elaboração de modelos específicos de análise - organização e categorização específicas;

- Diagnóstico das características dos estudantes, possibilidades, motivações, limitações e desenvolvimento socioemocional, técnico-formal e rítmico-expressivo para consequente prescrição de tarefas de superação e de estratégias de intervenção conducentes ao sucesso pedagógico;

- Planificação de intervenções pedagógicas assente num modelo misto, por objetivos e por projeto;

- Construção de programas considerando a integração de diferentes tipos de população alvo, diferentes graus de ensino e de diversidade de atividades específicas a cada população;

- Aplicação e controlo de técnicas, instrumentos e métodos de diagnóstico, controlo, aferição e reestruturação das aprendizagens específicas do processo de ensino do docente, da aprendizagem (cognitiva, socioafetiva e psicomotora) dos estudantes e da interação professor-aluno.

Centrada na construção de um projeto de intervenção de dança em contexto, a unidade curricular de MED propõe aos estudantes-professores vivenciar um ciclo de aprendizagem, planificação - intervenção - controlo - avaliação - reformulação da atividade pedagógica (LEBRUN; BERTHELOT,
1994), aproximando-se do Ciclo de Aprendizagem de Kolb (1984), fundador da Experience Learning Based Systems (2021). Sendo um modelo dinâmico de representação da aprendizagem das pessoas, atribui grande importância ao papel da experiência. Segundo Kolb (2015), as experiências imediatas ou concretas são a base para observações e reflexões. Num ciclo, identificado por este autor como ciclo de aprendizagem experiencial, estas reflexões são assimiladas e convertidas em conceitos abstratos dos quais se podem extrair novas implicações para a ação, que por sua vez podem ser experimentadas ativamente e servir como orientações na criação de novas experiências. Segundo McLeod (2017), o modelo de aprendizagem da teoria da aprendizagem experimental de Kolb preocupa-se com os processos cognitivos internos do estudante. Kolb (2015) explica que considera dois modos de entendimento, um Continuum de Perceção (a nossa resposta emocional ou como pensamos) entre a Experiência Concreta (EC) e a Conceção Abstrata (CA), e dois modos de transformação, um Continuum de Processamento (como nos aproximamos de uma tarefa) entre a Observação Reflexiva (OR) e a Experimentação Ativa (EA). Assim, a aprendizagem surge a partir da tensão criativa entre estes quatro modos de aprendizagem, Experienciar, Refletir, Conceptualizar e Aplicar. Segundo Kolb (2015), este processo deve ser entendido como um ciclo ou espiral de aprendizagem idealizada onde o aprendente experimenta, reflete, pensa e age, de acordo com a situação e conteúdo de aprendizagem.

A Experiência Concreta contempla o Experienciar, um entendimento via apreensão, enquanto a Conceção Abstrata refere-se ao Conceptualizar, um entendimento via compreensão. Quanto aos dois modos de transformação, a Observação Reflexiva contempla o Refletir, uma transformação via intenção, enquanto a Experimentação Ativa refere-se ao Aplicar ou Testar, uma transformação via extensão.

Ainda os mesmos autores, Kolb e Kolb (2013), defendem que a aprendizagem profunda alcança-se e torna-se integrativa e orientada para o desenvolvimento, quando se desenvolve num processo ho- 
lístico e envolve os quatro modos de aprendizagem: Experienciar, Refletir, Conceptualizar e Aplicar. É precisamente esta aprendizagem experiencial, reflexiva, conceptual e aplicativa que MED habilita e otimiza através do planeamento e da concretização de aulas no exercício de prática pedagógica.

\section{A Planificação Pedagógica}

O estabelecimento de objetivos na planificação pedagógica corresponde ao início do ciclo em que o estudante, com o intuito de aplicar as suas ideias, desenvolve atividades para conhecer o contexto e para intervir em dança munido de conhecimentos apreendidos ao longo da licenciatura. Para isso, o estudante avalia, planeia e cria a partir do conhecimento que detém do envolvimento em que intervirá, começando por definir objetivos (psicomotores, sócio afetivos ou cognitivos) e conteúdos, organizados em atividades, para a sua intervenção.

Para conhecer a população alvo, o estudante-professor recolhe informação junto de diferentes intervenientes (docentes, discentes, responsáveis da instituição, familiares) e socorre-se de diversificadas formas e instrumentos de recolha (questionário, observação de aulas, observação de métodos de ensino, dossiês individuais e/ou grupais, de entre outros). Deste modo, vivencia uma experiência concreta e obtém conhecimentos específicos sobre a realidade educativa alvo da sua futura intervenção, refletindo sobre a experiência vivenciada e conceptualizando/ aferindo sobre os objetivos e conteúdos definidos. Posteriormente cria e estrutura as atividades de dança de acordo com a sua adequação às temáticas em desenvolvimento, à consecução dos objetivos gerais e específicos, sujeitando-as a avaliação formativa no decorrer do processo ensino-aprendizagem. Importa estabelecer, de forma coesa e coerente a conexão existente entre objetivos - conteúdos - atividades - avaliação clarificando o que o estudante-professor preconiza para a sua intervenção educativa. Podemos, então, afirmar que a planificação pedagógica funciona como um contrato de realização do projeto de intervenção em dança, onde se fixa a produção esperada, se decide a conclusão temporal e se avalia a conceção do projeto (DESSUS, 2006). Estabelecem-se relações com os objetivos, organizam-se etapas, instituem-se tarefas e tempos de aprendizagem e formalizam-se processos.

$\mathrm{Na}$ planificação todas as atividades que são idealizadas e estruturadas assumem forma e conteúdo com um nível de explicitação de acordo com o grau de maturação do estudante, devendo adequar-se à população alvo, aos objetivos e às temáticas a trabalhar. O estudante-professor deve ainda conceptualizar como avaliar (avaliação diagnóstica, formativa, sumativa e/ou prognóstica) (RIBEIRO; RIBEIRO, 2003) a intervenção a que se propõe, nomeadamente ao nível da tríade:

1. objetivos do programa - conteúdos de aprendizagem;

2. conteúdos de aprendizagem - instrumentos de avaliação;

3. objetivos do programa - instrumentos de avaliação.

Na planificação e estruturação das atividades há que pensar o modo como serão ministradas (decisões pré-ativas do professor de dança), bem como associar os estilos de ensino, os modelos de intervenção e as estratégias de comunicação mais adequados à concretização das atividades, e sempre de acordo com as características do grupo, em geral, e de cada aluno em particular (ALVES; MOURA, 2020).

\section{Prática Pedagógica}

Após a elaboração da planificação por parte dos estudantes-professores, orientada e tutorada pelos docentes de MED, dá-se início ao exercício de prática pedagógica que se constitui como uma estrutura programática desta abordagem educativa. O exercício da prática pedagógica compreende a conceção e a subsequente aplicação de planos de aula, seguida da posterior elaboração de relatórios de aula onde se consuma a autoavaliação, a avaliação recíproca (entre colegas observadores de aula) e a avaliação formativa (dos docentes de MED) realizadas através de debates em contexto de sala de aula e de fichas de 
observação das aulas lecionadas. Nesta etapa, podemos considerar uma aproximação ao conceito de ciclo de aprendizagem experiencial de Kolb (1984) ao atentar à ordenação: experiência concreta de lecionação de uma aula real, consequente reflexão, congruente conclusão formalizada no relatório, e nova adaptação para o plano de aula seguinte (figura 1). Esta ordem de atividades ou espiral de experienciação permite condições efetivas, para que cada estudante descubra e/ou aprofunde o que é "ser professor".

Figura 1. Adaptação do ciclo de aprendizagem experiencial de Kolb às atividades da unidade curricular de Metodologias do Ensino da Dança.

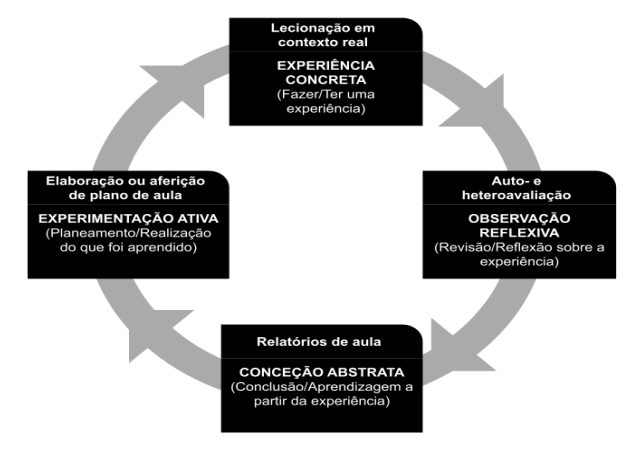

Pretende-se que cada estudante encontre o seu próprio estilo de "ser professor", não obstante, este modelo de formação implica necessariamente conhecer com profundidade o ato pedagógico, assim como dominar técnicas, métodos e estratégias de ensino. Também, a avaliação da experiência de ensino e de aprendizagem que cada estudante-professor faz depende do estilo individual na adequação às condições concretas de exercício da atividade docente. Concomitantemente a avaliação do estudante-professor, realizada pelos docentes de MED, assume-se formativa, estimulando as potencialidades individuais dos formandos, durante todo o processo e sumativa, com funções formativas, aquando da entrega do relatório final do exercício de prática pedagógica. Deste modo, os estudantes participam ativa e colaborativamente nas atividades de Ensino, Observação, Avaliação e Reensino.
Na conceção do plano de aula, e dando especial atenção à adequabilidade do mesmo relativamente às características da população alvo, aos objetivos da aula e à temática, os estudantes-professores enunciam os objetivos comportamentais que querem trabalhar na aula e descrevem, quer o comportamento do professor (que instrução transmite, como transmite, que ações e atitudes prevê utilizar) quer as atividades dos alunos. Na descrição das atividades (objetivos operacionais da aula) definem o comportamento desejável, as condições de realização e os critérios de êxito que os alunos deverão conseguir apresentar. Para a consecução das atividades e alcance dos objetivos propostos para a aula, definem as estratégias e métodos de instrução que consideram adequados, tendo em conta as 4 dimensões de ensino - Instrução e Feedback, Gestão, Disciplina e Clima de aula -; sugerem e organizam recursos materiais e humanos; subdividem, sequenciam e estruturam as atividades pelas fases da aula (Início, Desenvolvimento, Clímax e Final) atribuindo durações específicas para cada fases de aula e para cada atividade.

Durante a lecionação de aulas, os estudantes-professores vivenciam a sua experiência de lecionarem uma aula real, com uma população concreta e específica, registam os colegas de turma observam, registam e avaliam a lecionação em curso, em fichas de observação de aulas reais, onde constam as 4 dimensões de ensino (Instrução e Feedback, Gestão, Disciplina e Clima de aula) ou, alternativamente, em fichas de observação de aulas de microensino - aulas em que o estudante-professor se preocupa em melhorar e aperfeiçoar a dimensão de ensino menos conseguida na aula real -. Neste caso, a observação incide sobre a dimensão assumida na lecionação de microensino.

Após a lecionação, os estudantes-professores elaboram os relatórios das aulas, onde inscrevem a sua própria avaliação e comparam-na com a dos seus pares e com a avaliação formativa proveniente dos docentes de MED. Nesta fase, têm também oportunidade de relacionar os planos de aula 
(elaboração e aplicação) com a planificação pedagógica desenhada, de processar os imprevistos e suas reações, e de tecer considerações futuras a inscrever nos próximos planos de aula, concluindo deste modo o processo de prática pedagógica.

Consequente da estruturação e aplicação programática operada em MED as competências adquiridas relacionam-se com a capacitação do estudante para o ensino/formação em dança. Quando concluída a unidade curricular de MED, o estudante-professor:

- Planifica, estrutura e idealiza projetos de intervenção pedagógica tangíveis;

- Define e relaciona objetivos educativos com os conteúdos programáticos, as atividades de aprendizagem e a avaliação de processo, específicos a práticas pedagógicas concretas;

- Adequa o conteúdo específico de dança a diversificadas populações alvo (diferentes escalões etários, níveis de proficiência, motivações e interesses);

- Demonstra conhecimento didático do conteúdo no âmbito de diferentes formas de dança e respetivas progressões na aprendizagem;

- Constrói, fundamenta e aplica planos de aula específicos a diferentes formas de dança, em vários contextos de intervenção e de acordo com: as temáticas das aulas, os objetivos a alcançar, os conteúdos programáticos, as atividades concretas, as estratégias e métodos de comunicação e a avaliação da aula.

\section{Conclusões}

A vivência de processos educativos no âmbito de metodologias do ensino da dança, possibilita a oportunidade de experienciação e vivência de situações novas no domínio do ensino da dança e das práticas expressivas no sistema de ensino e em contexto comunitário, de animação e lazer. O estudante concebe, planeia, aplica, controla, avalia e reformula situações de ensino e de aprendizagem da dança, orientadas por objetivos e por projeto numa relação dialética e complementar.
Ao defendermos a utilização de uma pedagogia mista de trabalho por objetivos e por projeto, enaltecemos as valências pedagógicas, criativas e de investigação daí advindas, onde o valor dos resultados dos alunos é consequência de uma multiplicidade de fatores, "a multiplicidade é inevitável numa sociedade pluralista” (SACRISTÁN, 1990, p. 92). Temos de considerar quer etapas de aprendizagem sequenciais, estruturadas, observáveis e mensuráveis, quer a diversidade dos comportamentos, das ações e dos pensamentos dos estudantes, dos seus processos subjetivos individuais, e grupais, socialmente verdadeiros e colaborativos.

Aos docentes cabe a mentoria, tutoria e avaliação de todo o processo de ensino e de aprendizagem dos seus estudantes facilitando, na análise, na forma e no conteúdo, a descoberta das soluções mais adequadas às diferentes etapas e tarefas de aprendizagem orientadas para várias populações alvo e para diferenciados contextos educativos.

A Metodologia do Ensino da Dança ao estimular o estudante para a participação nos processos de conceção, organização, intervenção e controlo de projetos educativos com população real, tutorados pelo docente, bem como o contacto com outros profissionais de ensino, com diferentes características, formação e métodos, contribui de forma decisiva para elevar o conhecimento, a experiência e a formação do licenciado em dança.

\section{Referências}

ALVES, M. J.; MOURA, M. Estratégias para o Ensino e Aprendizagem de Dança Strategies for Teaching and Learning Dance. Revista Portuguesa de Educação Artística, v. 10, n. 1, p. 7-32, 2020.

EXPERIENCE BASED LEARNING SYSTEMS, LLC. Disponível em: https://learningfromexperience.com/ research-library/experiential-learning-theory-bibliography-volume-6/. Acesso em: 25 fev. 2021. 
DESSUS, P. Quelles idées sur l'enseignement nous révèlent les modèles d'Instructional Design? Revue Suisse des Sciences de l'Éducation, v. 28, n. 1, p. 137-157, 2006.

DEWEY, J. Vida e educação. 10a ed. São Paulo: Melhoramentos, 1978.

KOLB, A. Y.; KOLB, D. A. The Kolb Learning Style Inventory 4.0. A Comprehensive Guide to the Theory, Psychometrics, Research on Validity and Educational Applications. [s.I.] Experience Based Learning Systems, 2013. Disponível em: https://learningfromexperience.com/research-library/the-kolb-learning-style-inventory-4-0/. Acesso em: 1 mar. 2021.

KOLB, D. A. The process of experiential learning. In: KOLB, D. A. (Org.). The experiential learning: Experience as the source of learning and development. New Jersey: Prentice-Hall. 1984. cap. 2, p. 20-38.

KOLB, D. A. Experiential learning: Experience as the source of learning and development. 2nd. ed. New Jersey: Pearson Education, 2015.

KILLEN, R. Effective teaching strategies: Lessons from research and practice. 7. ed. South Melbourne:

Cengage Learning, 2016.

LEBRUN, N.; BERTHELOT, S. Plan pédagogique: une démarche systématique de planification de l'enseignement. Bruxelles: De Boeck Université, 1994.

MCLEOD, S. A. Kolb's learning styles and experiential learning cycle | Simply psychology. Disponível em: https://www.simplypsychology.org/learning-kolb. html. Acesso em: 25 fev. 2021.

NIGHTINGALE, P.; O'NEIL, M. Achieving quality learning in higher education. London: Routledge, 1994.
PINTO, M. G. Dos construtos teóricos para as aplicações: o professor como um dos mediadores. Revista de Estudos Linguísticos da Universidade do Porto, v. 1, n. 6, p. 93-123, 2011.

RIBEIRO, A. C.; RIBEIRO, L. C. Planificação e Avaliação do Ensino-Aprendizagem. Lisboa: Universidade Aberta, 2003.

SACRISTÁN, J. La pedagogía por objetivos: Obsesión por la eficiencia. Madrid: Ediciones Morata, 1990.

WARBURTON, E. C. Beyond steps: The need for pedagogical knowledge in dance. Journal of Dance Education, v. 8, n. 1, p. 7-12, 2008.

VASCONCELOS, T. Trabalho de Projeto como "Pedagogia de Fronteira". Da investigação às práticas, v. 1, n. 3, p. 8-20, 2011.

Recebido: 09/03/2021

Aceito: $12 / 04 / 2021$

Aprovado para publicação: 28/05/2021

Este é um artigo de acesso aberto distribuído sob os termos de uma Licença Creative Commons Atribuição 4.0 Internacional. Disponível em: $<$ http://creativecommons.org/licenses/by/4.0>.

This is an open-access article distributed under the terms of the Creative Commons Attribution License 4.0 International. Available at: <http://creativecommons.org/licenses/by/4.0>.

Ce texte en libre accès est placé sous licence Creative Commons Attribution 4.0 International. Disponible sur: <http://creativecommons.org/licenses/by/4.0>. 